\title{
Annotation and characterization of the Plasmodium vivax rhoptry neck protein 4 (PvRON4)
}

Gabriela Arévalo-Pinzón ${ }^{1,2}$, Hernando Curtidor ${ }^{1,2}$, Jesica Abril ${ }^{1}$ and Manuel A Patarroyo , 2* $^{*}$

\begin{abstract}
Background: The tight junction (TJ) is one of the most important structures established during merozoite invasion of host cells and a large amount of proteins stored in Toxoplasma and Plasmodium parasites' apical organelles are involved in forming the TJ. Plasmodium falciparum and Toxoplasma gondii apical membrane antigen 1 (AMA-1) and rhoptry neck proteins (RONs) are the two main TJ components. It has been shown that RON4 plays an essential role during merozoite and sporozoite invasion to target cells. This study has focused on characterizing a novel Plasmodium vivax rhoptry protein, RON4, which is homologous to PfRON4 and PkRON4.

Methods: The ron4 gene was re-annotated in the P. vivax genome using various bioinformatics tools and taking PfRON4 and PkRON4 amino acid sequences as templates. Gene synteny, as well as identity and similarity values between open reading frames (ORFs) belonging to the three species were assessed. The gene transcription of pvron4, and the expression and localization of the encoded protein were also determined in the VCG-1 strain by molecular and immunological studies. Nucleotide and amino acid sequences obtained for pvron4 in VCG-1 were compared to those from strains coming from different geographical areas.

Results: PVRON4 is a 733 amino acid long protein, which is encoded by three exons, having similar transcription and translation patterns to those reported for its homologue, PfRON4. Sequencing PVRON4 from the VCG-1 strain and comparing it to $P$. vivax strains from different geographical locations has shown two conserved regions separated by a low complexity variable region, possibly acting as a "smokescreen". PvRON4 contains a predicted signal sequence, a coiled-coil a-helical motif, two tandem repeats and six conserved cysteines towards the carboxyterminus and is a soluble protein lacking predicted transmembranal domains or a GPI anchor. Indirect immunofluorescence assays have shown that PvRON4 is expressed at the apical end of schizonts and co-localizes at the rhoptry neck with PVRON2.
\end{abstract}

Conclusions: Genomic, transcriptional and expression data reported for PVRON4, as well as its primary structure characteristics suggest that this protein participates in reticulocyte invasion, as has been shown for its homologue PARON4.

Keywords: Bioinformatics analysis, Invasion, Plasmodium vivax, Rhoptry neck protein, Rhoptry, Tight junction

\section{Background}

Malaria is a parasitic disease, primarily affecting pregnant females and children aged less than five years in tropical and subtropical areas around the world, mainly in sub-Saharan Africa [1]. A substantial increase in cases of Plasmodium vivax malaria has been reported recently in Asia and Latin America [2]. Such data, added to an increase in the number of severe disease reports, death

\footnotetext{
* Correspondence: mapatarr.fidic@gmail.com

${ }^{1}$ Fundación Instituto de Inmunología de Colombia FIDIC, Carrera 50 \# 26-20, Bogotá, Colombia

${ }^{2}$ Universidad del Rosario, Carrera 24 \# 63C-69, Bogotá, Colombia
}

and resistance to first-generation anti-malarial drugs attributed to $P$. vivax [3-5], emphasize the need for developing effective control tools and acknowledging this parasite species as an important agent of vectortransmitted tropical diseases.

Basic research around the world into $P$. vivax has lagged behind compared to Plasmodium falciparum mainly due to a lack of in vitro continuous culturing as this requires a large amount of reticulocytes for maintaining and propagating this parasite species [6]. Among the various strategies being used to overcome this problem, comparative

\section{( Biomed Central}

(c) 2013 Arévalo-Pinzón et al.; licensee BioMed Central Ltd. This is an open access article distributed under the terms of the Creative Commons Attribution License (http://creativecommons.org/licenses/by/2.0), which permits unrestricted use, distribution, and reproduction in any medium, provided the original work is properly cited. 
analysis with other species, such as $P$. falciparum and adapting $P$. vivax strains to Aotus monkeys [7], have allowed the identification during the last few years of several antigens in $P$. vivax which could be participating in invasion [8-11].

The merozoite's rapid and coordinated invasion of red blood cells $(\mathrm{RBC})$ is mediated by initial contact with the host cell, re-orientation of the parasite's apical pole and active internalization into the host cell involving many sub-compartmentalized proteins localized in secretory organelles, such as micronemes and rhoptries [12]. The rhoptries are pear-shaped structures having two compartments called neck and bulb; despite being present in all parasites belonging to the phylum Apicomplexa, their size and number varies according to the target cell being invaded [13]. Transmission electron microscopy studies of $P$. falciparum and Toxoplasma gondii have revealed that some antigens are site-specifically distributed in a rhoptry $[14,15]$; such distribution seems to be the rule concerning important organization facilitating protein function in tight junction (TJ) and/or parasitophorous vacuole (PV) formation. Proteomics analysis of $T$. gondii rhoptries has shown minimum overlapping with $P$. falciparum proteins, except for some proteins localized in the rhoptry neck (RON), which could be involved in functions common to both parasites [16]. RON2 [17], RON4 [18] and RON5 [19] proteins have been identified to date in P. falciparum, forming part of a high molecular weight complex interacting with a microneme-derived protein called apical membrane antigen 1 (AMA-1) [20]. The assembly of this macromolecular complex allows TJ formation or irreversible binding which is important since this acts as a foothold for the parasite's motor machinery to propel the parasite into the PV for successful invasion.

Surface plasmon resonance (SPR) studies have determined that the PfRON2 binding region to PfAMA-1 lies within residues 2,020-2,059 and contains two conserved cysteines which are strongly implicated in the peptide's structure [21]. Crystallization of the PfAMA-1/PfRON2 complex has shown that the peptide binds in a U-shape to the AMA-1 ectodomain, inducing a conformational change in AMA-1 domain II (DII) [21], similar to that found for the interaction between $\operatorname{TgAMA}-1 / \operatorname{Tg} \mathrm{RON} 2$ [21]. Interestingly, different methodological approaches have proposed that blocking the interaction between AMA-1 and RON2 inhibits parasite invasion of its host cell [20,22,23]. Knowledge regarding these interactions has been key in understanding 4G2 antibody's invasioninhibitory action (binding to AMA-1 DII), thereby impeding displacement in domain II and thus blocking interaction with RON2 [21].

PfRON4 is the second most studied antigen from the aforementioned complex after AMA-1 and RON2. This is one of the most important proteins as it is most likely essential in merozoite and sporozoite invasion of their respective host cells [24]. No evidence has been advanced to date regarding the role played by RON4 in TJ formation and it is not known which proteins interact directly with it within the complex. However, RON4 has been the only protein from the whole complex that has been convincingly localized to junction constriction during invasion [25]. In fact, immunoprecipitation assays with Plasmodium yoelii merozoite proteins have supported RON4 (PyP140)-AMA1 interaction but PyRON2 and PyRON5 have not been identified within the complex. Importantly, immunogenicity studies have shown that PfRON4 sequences elicit immunogenic responses in natural human malaria infection, suggesting their exposure to the immune system during host cell invasion as has been proposed for other important antigens, such as merozoite surface proteins (MSP) [26].

The importance of RON proteins in P. falciparum and in the model Apicomplexa parasite, T. gondii, has led to interest in identifying the functional characterization of RON proteins in other genera, such as Neospora, Cryptosporidium, Babesia, and other Plasmodium species $[8,27]$. Recent studies have shown that although most proteins from the complex are conserved within Apicomplexa, some are exclusive to certain members [27]. Such divergence regarding the complex's molecular composition suggests differences in TJ formation among Apicomplexa members and partly explains host cell specificity among parasites [27].

This study was thus aimed at making a correct annotation for pvron 4 in the P. vivax genome and characterizing PvRON4 in late-stage schizonts from the VCG-1 strain. PvRON4 is a 733 amino acid long protein containing typical characteristics concerning antigens involved in invasion, such as having a signal peptide, coiled-coil $\alpha$-helical motifs, low complexity regions, and long conserved segments. Immunofluorescence studies using rhoptry and membrane markers as reference have shown that $P v$ RON4, just like its homologue in P. falciparum (PfRON4), is localized in the rhoptries and could thus be participating in invasion.

\section{Methods \\ Bioinformatics tools}

The Basic Local Alignment Search Tool (BLAST), from the National Center for Biotechnology Information (NCBI), was used to find the homologous gene to pfron4 (PF11_0168) and pkron4 (PKH_091340) in the P. vivax genome. The presence of and boundaries between pvron 4 introns and exons was evaluated using Genscan [28], Spidey [29] and tBlastn. Gene structure, open reading frame (ORF) transcription direction, nucleotide and amino acid level identity, and similarity values between 
P. falciparum-P. vivax and Plasmodium knowlesi-P. vivax were taken into account for synteny analysis.

Bioinformatics tools, such as SignalP [30], were used for evaluating the presence of a signal peptide in PvRON4 sequence; TMHMM, Polyphobius and PredGPI were used to predict transmembranal domains and glycosylphosphatidylinositol (GPI) anchors, since the presence of these motifs and domains is typical of several antigens considered as candidates for an antimalarial vaccine [31-33]. Sequence tandem repeats extraction and architecture modelling (XSTREAM, variable ' $\mathrm{X}$ ') was used for finding repeat sequences and the simple modular architecture research tool (SMART) [34] with Globplot for searching for other important motifs and domains.

\section{DNA, RNA and protein source and CDNA synthesis}

A blood sample taken from an Aotus sp. monkey infected with the Colombian $P$. vivax Guaviare 1 strain (VCG-1) was used for extracting nucleic acids and proteins. The VCG-1 strain had been cultured via successive passes in vivo in Aotus sp. monkeys from the primate station in Leticia, Amazonas, as thoroughly described [7]. The monkeys were kept under constant care, supervised by a primatologist, according to the conditions established by Colombian Animal Protection law (law 84/1989) and Corpoamazonía (the Colombian entity regulating environmental matters in the region) (resolution 00066, September 13, 2006).

A schizont-enriched sample was obtained using a discontinuous Percoll gradient (GE Healthcare) and genomic DNA (gDNA) was isolated using a Wizard genomic DNA purification kit (Promega), following the manufacturer's recommendations. RNA was isolated from the parasite using the Trizol method [35], followed by treatment with RQ1 RNase-free DNase (Promega). Purified RNA and gDNA integrity was examined by electrophoresis on agarose gels. Five microlitres of RNA were taken as template for cDNA synthesis, using a onestep RT-PCR SuperScript III kit (Invitrogen).

\section{Primer design and pvron4 amplification}

Based on bioinformatics analysis, the PVX_091435 gene ID gene was re-annotated (Figure 1) and such information was used to design primers for amplifying pvron4 from gDNA and cDNA. pvron4G1 5'- ATG TCT CGT AAA AGG GTT TT-3' and pvron4G2 5'-CAA GTC TTC AAA AAT GAG ATT T-3' primers amplified from predicted ATG until the stop codon. These primers were included in PCR reactions with Gotaq flexi DNA polymerase (Promega) with gDNA or RT-PCR products from samples treated with or without reverse transcriptase $\left(\mathrm{RT}^{+}\right.$ and $\mathrm{RT}^{-}$respectively) at $25 \mu \mathrm{L}$ final volume, according to the manufacturer's instructions. Amplification conditions were as follows: a 5 -min cycle at $95^{\circ} \mathrm{C}$, followed by 35 cycles of $1 \mathrm{~min}$ at $56^{\circ} \mathrm{C}, 3 \mathrm{~min}$ at $72^{\circ} \mathrm{C}$ and $1 \mathrm{~min}$ at $95^{\circ} \mathrm{C}$. A final extension step lasted $10 \mathrm{~min}$ at $72^{\circ} \mathrm{C}$. A pvron 1 region ( 1053 from cDNA) transcribed during the erythrocyte phase was amplified using direct $5^{\prime}$ - atg GCG AAG GAG CCC AAG TG-3' and reverse 5' - ATC CCT AGC AAT GCT TCG -3' primers for evaluating cDNA contamination by gDNA [36].

Primers called ExtD: 5'-TCC AGA CGT GTC AGA GTG-3' and ExtR: 5'- TAG CTT CGT TCC TTT GGG3 ' were also designed; they were localized 72 bp upstream of the start codon and $49 \mathrm{bp}$ downstream the stop codon of the pvron 4 gene, respectively. Amplification conditions from gDNA and cDNA were the same as those used for the pvron $4 \mathrm{G} 1$ and pvron $4 \mathrm{G} 2$ primers. The polymerase chain reaction (PCR) products were visualized on $1 \%$ agarose gel and then purified with a Wizard PCR preps kit (Promega). PCR products from gDNA and cDNA were cloned in pGEM-T (Promega) and $\mathrm{pEXP5}-\mathrm{CT} / \mathrm{TOPO}$ vectors (Invitrogen) respectively by TA cloning. Positive clones and PCR products were sequenced in an ABI PRISM 310 Genetic Analyzer. The sequences were analyzed using CLC DNA Workbench (CLC bio) and compared to the Sal-1, Mauritania I and India VII strain sequences [37] using Clustal W [38].

\section{Obtaining polyclonal antibodies against PvRON4, Pv12 and PvRON2}

Taking the amino acid sequence predicted for PvRON4 as reference, two 20 residue-long, B-epitope peptides were selected, chemically synthesized in solid phase and cysteine and glycine (CG) were added to amino and carboxy termini for polymerization. Synthetized peptides were numbered 36114 (CG ${ }^{181}$ GSASESAAPSEEKASEE $\left.\mathrm{NVP}^{200} \mathrm{GC}\right)$ and 36115 (CG ${ }^{719}$ AAEVEKRGFDEDYIQEEI $\mathrm{KN}{ }^{738} \mathrm{GC}$ ). These peptides were selected based on high average values for Parker's antigenicity, hydrophilicity and solvent accessibility using Antheprot software [39] and high values obtained when using Bepipred (0.35 default threshold and $75 \%$ specificity) [40]. It was also taken into account that the peptides corresponded to different protein regions, for detecting fragments in case of proteolytic processing occurring in PvRON4.

Seven to eight week-old BAlb/C mice were intraperitoneally (ip) inoculated with $75 \mu \mathrm{g}$ of each polymeric peptide emulsified in Freund's complete adjuvant (FCA) on day 0 for evaluating PvRON4 expression in VCG-1 strain schizonts. Three boosters were given on days 30 , 45 and 60 using the same peptides emulsified in Freund's incomplete adjuvant (FIA). Polyclonal antibodies against $P v 12$ localized on the membrane and $P v$ RON2 localized in the rhoptries were obtained for PvRON4 localization studies. Peptide 35520 (CG ${ }^{1674}$ KLQQEQNELNEEKERQ 


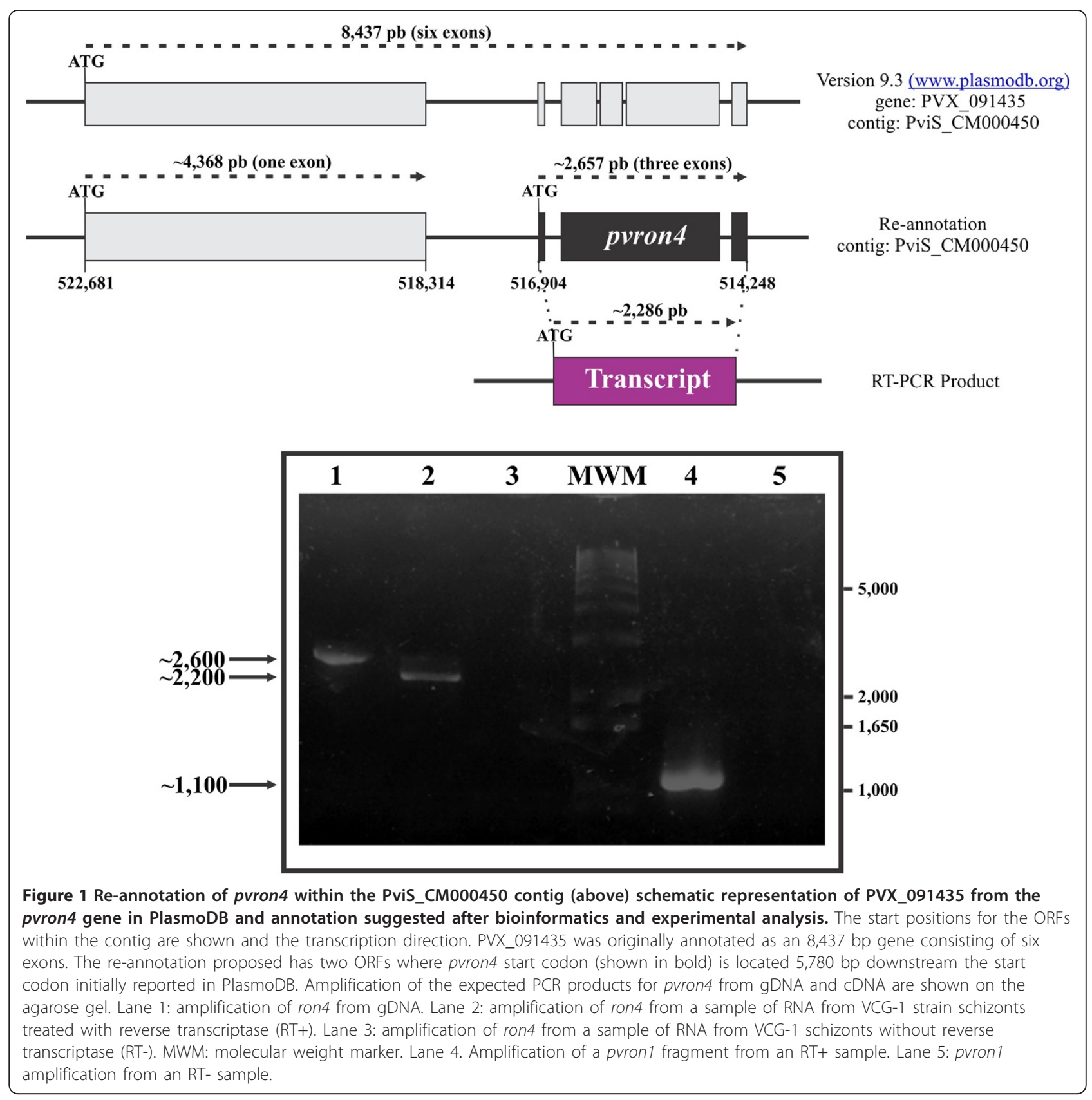

RQEN $^{1693} \mathrm{GC}$ ) [8] was used for PvRON2 and peptides CG-AKIRVRKRSGEEYDKEIFNL-GC and CG-AHFEFA TTPDDQNSVSEPRA-GC were used for Pv12 [10]. These peptides were inoculated into negative New Zealand rabbits for recognizing $P$. vivax-derived proteins by Western blot, as described previously $[8,10]$. Animal sera was collected and stored at $-70^{\circ} \mathrm{C}$ for later studies. Animal immunization and bleeding was done according to Colombian animal protection recommendations (Law 84/1989) and Resolution 8430/ 1993 for handling live animals for research or experimentation purposes.

\section{PvRON4 immunodetection assays \\ ELISA}

A previously described ELISA test was used for determining inoculated peptide immunogenicity [19]. Briefly, each of the inoculated peptides was sown in 96-well ELISA plates and incubated at 1:100 dilution of each serum. A peroxidase-coupled, anti-mouse antibody was used as secondary antibody (Vector Laboratories), diluted 1:5,000.

\section{Western blot}

The proteins were extracted from a $P$. vivax schizontenriched sample and separated on an SDS-PAGE gel, as 
previously described [8]. Proteins transferred to a nitrocellulose membrane were incubated with pre-immune or immune serum. In a parallel assay, immune serum was pre-incubated with inoculated polymeric peptides before being incubated with the nitrocellulose membrane. A peroxidase-coupled, anti-mouse IgG antibody was used as secondary antibody (Vector Laboratories).

\section{Indirect immunofluorescence}

A suspension of erythrocytes parasitized by $P$. vivax VCG-1 was washed thrice with PBS ( $\mathrm{pH} 7.2-7.4$ ), then centrifuged at 2,500 $\times \mathrm{g}$ for $4 \mathrm{~min}$ and suspended in a 1:1 PBS-fetal bovine serum solution. This solution was seeded on eight-well multitest slides and left to dry at room temperature for $24 \mathrm{hr}$. Parasites slides were fixed using a $4 \%$ formaldehyde solution followed by three washes with PBS. The slides were blocked with $1 \%$ skimmed milk in PBS for $30 \mathrm{~min}$ and incubated with anti-PvRON4 primary antibody obtained in mice and anti-Pv12 or anti-PvRON2 antibodies in 1:40 dilution. Fluorescein-labelled anti-rabbit IgG (FITC) (Vector Laboratories) and rhodamine-labelled anti-mouse IgG (Millipore) were used as secondary antibody for $60 \mathrm{~min}$, followed by three PBS washes. Parasite nuclei were stained with a $2 \mu \mathrm{g} / \mathrm{mL}$ solution of 4,6-diamidino-2phenylindole (DAPI) for $20 \mathrm{~min}$ at room temperature and fluorescence was visualized in a fluorescence microscope (Olympus BX51) using an Olympus DP2 camera and Volocity software (Perkin Elmer).

\section{Statistical analysis}

Differences in antibody production between pre-immune and post-third immunization sera obtained from ELISA were evaluated using non-parametric Wilcoxon signed rank test.

\section{Results and discussion In-silico re-annotation of the ron4 gene in Plasmodium vivax}

The search for the pvron 4 gene was initially made using the PfRON4 protein amino acid sequence (PF11_0168) containing 1,201 residues [18]. tBlastn results revealed a nucleotide region having a high probability of containing pvron4 in the PviS_CM000450 contig localized in chromosome 9. Analysis showed a pvron4 segment running from nucleotide 516,555 to 514,248 corresponding to residues 484 to 1,201 in $P$. falciparum; however, the beginning of prron4 could not be found. The P. knowlesi RON4 (PKH_091340) amino acid sequence reported in PlasmoDB was used to overcome this. Plasmodium knowlesi is a parasite which infects Macaca fascicularis monkeys and shares a close phylogenetic relationship with $P$. vivax as shown in small subunit rRNA analysis and the high identity and similarity values between orthologous proteins $[9,41]$. This led to finding that pvron4 was located between nucleotide 516,904 and 514,248 , having a $\sim 2,657$ bp length. Interestingly, a putative gene called PVX_091435 (8,437 bp and six exons) was found to be annotated in this nucleotide region which is also found when PfRON4 and PkRON4 amino acid sequences are used as templates for Blastp analysis (Figure 1). However, analyzing the presence of and boundaries for the exons/introns proposed for the PVX_091435 gene indicated that its gene structure had not been correctly annotated (Figure 1). In-silico analysis of this genome locus suggested that there were two ORFs; the first had high similarity $(60 \%)$ with a $P$. falciparum serine esterase and began at position 522,681 of the PviS_CM000450 contig and ended at nucleotide 518,314 (Figure 1) while the second ORF was found downstream serine esterase in position 516,904 and referred to pvron4. The suggested pvron4 structural region had three exons; a first short one $\sim 78$ bp (in ORF -1) having consensus intron splice sites, a second long $\sim 2,019$ bp (in ORF -2) having its respective donor site sequence ending with a 189 bp exon (in ORF -3) (Figure 1).

Once ron 4 structure had been annotated, a syntenic analysis was made for evaluating neighboring genes' structure (number of introns and exons), transcription orientation and determining identity (ID) and similarity (SI) values at protein level. PvRON4 amino acid alignment with PfRON4 and PkRON4 showed that ID and SI values were greater with PkRON4 (Figure 2) thereby agreeing with the close relationship between these two parasite species. This coincided with previous studies showing greater similarity between $P$. vivax and $P$. knowlesi proteins than with other Plasmodium species $[10,11]$. Conservation was also found regarding the structure of genes upstream and downstream ron 4 in $P$. falciparum, P. vivax and P. knowlesi, having 61.1\%-97\% and $77.6 \%-99.7 \%$ amino acid ID and SI values for $P k-P v$ respectively, and $33.6 \%-88 \%$ and $54 \%-100 \%$ for $P f-P v$, respectively (Figure 2).

\section{Transcription and analysis of the VCG-1 strain pvron4 sequence}

The pvron 4 gene was amplified from gDNA and cDNA to confirm its presence and re-annotation. Figure 1 shows that a $\sim 2,600$ bp gDNA product was obtained and a $\sim 2,200$ bp product from cDNA (RT+) thereby confirming the presence of intron regions. No amplification was observed from RT- samples, indicating that the cDNA preparation had no gDNA contamination. PCR amplification of the pvron4 encoding sequence suggested the presence of this transcript $(\sim 2,200 \mathrm{bp})$ in mature $P$. vivax blood stages, similar to that found for pvron 1 (Figure 1) [36]. The pvron4 transcription time coincided with that displayed by pfron 4 (42-48 hr of the intra- 


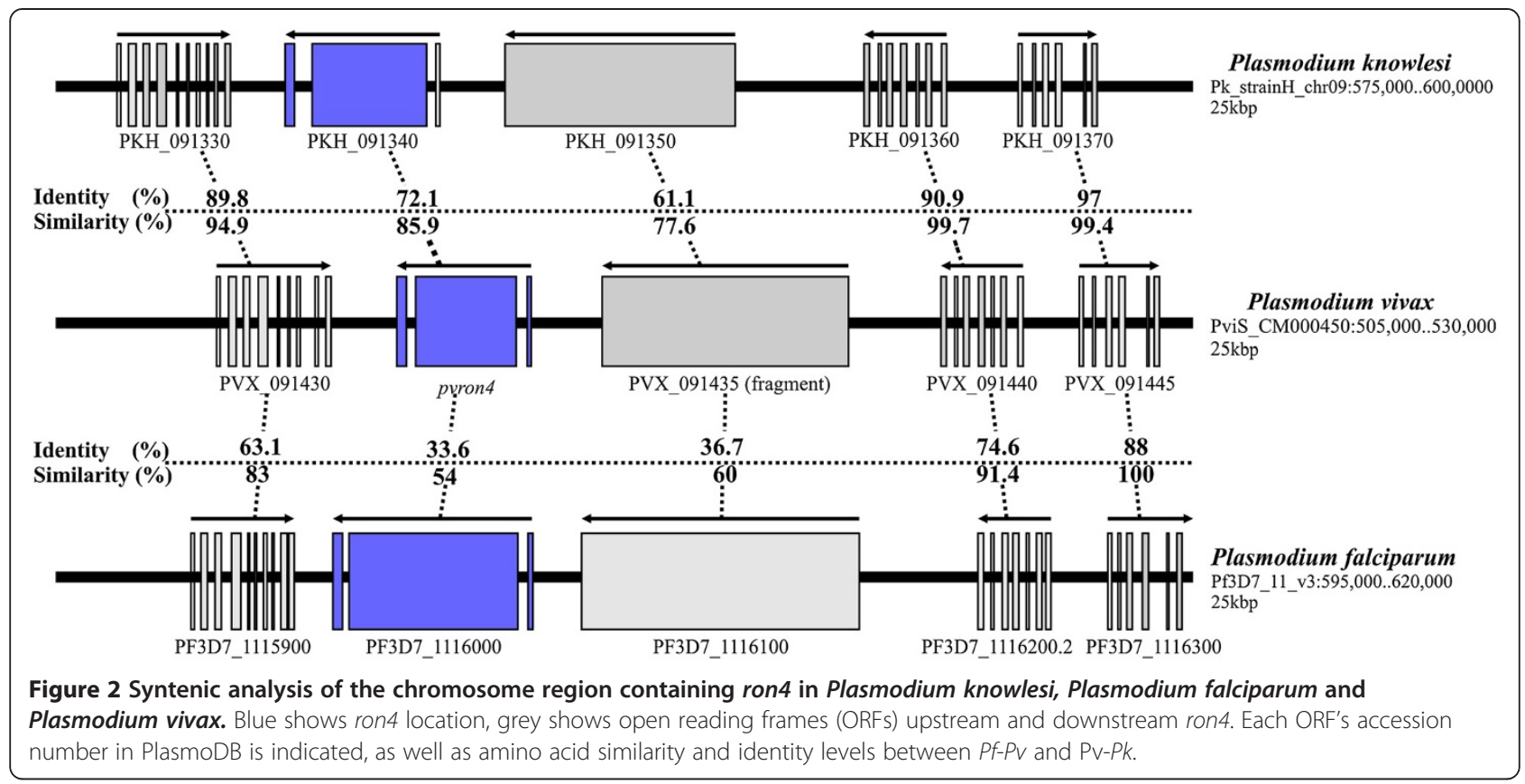

erythrocyte cycle) [42]. Transcriptome and proteome analysis of $P$. falciparum has shown that most ORFs transcribed at the end of the intra-erythrocyte cycle are expressed during and participate in host cell invasion $[42,43]$.

When aligning pvron 4 gDNA and cDNA nucleotide sequences obtained from sequencing three independent clones it was found that the gDNA was $2,573 \mathrm{bp}$ while cDNA was 2,202 bp. The gene consisted of three exons and two intron regions, encoding a 733 residue-long protein $(\sim 80 \mathrm{kDa})$; this genomic organization coincided with that proposed by bioinformatics analysis (Figure 1). When comparing the sequences obtained from VCG-1 to those reported for Sal-1 (PVX_091435), a match was found from nucleotide 516,904 onwards within the contig, coinciding with previous analysis proposed for pvron4 localization and structure (Figure 1). The alignment showed that exon 2 was shorter in VCG-1 $(1,932$ bp) compared to Sal-1 (2,019 bp) due to deletions corresponding to a loss of $81 \mathrm{nt}$ ( 27 amino acid residues) between amino acids 235-262 and a deletion of two triplets (GTG AGG) encoding glycine (G671) and glutamic acid (E672). According to bioinformatics analysis, exon two encoded a low complexity region rich in tandem repeats (TR) (Figure 3). Analysis also revealed a change at nucleotide level involving $T$ for $G$ in position 810 (T810G); this substitution led to a valine (V) being changed for a glycine (G) in position 199 (V199G). A substitution of $A$ for $G$ was found in VCG-1 strain intron 2 compared to Sal-1. VCG-1 gDNA and cDNA consensus sequences were deposited in [GenBank: KF378614 and KF378615].
Comparative analysis between VCG-1 PvRON4 (Colombian), Sal-1 (El Salvador), India VII (southern Asia) and Mauritania I (Africa) amino acid sequences revealed differences regarding protein length (see Additional file 1), mainly due to large deletions between residues 179-305 where the TRs occurred. The first 150 amino acids corresponded to a conserved region followed by a variable zone having repeat regions and a highly conserved carboxy-terminal in the strains evaluated here. Such distribution was similar to that reported for several antigens proposed as candidates for an anti- $P$. falciparum vaccine where proteins' conserved regions are surrounded by variable and low complexity regions acting as immune system distractors, while important functional regions for the parasite remain poorly recognized. The circumsporozoite protein (CSP) consists of a central repeat region, which is diverse across Plasmodium species, and flanking the repeats are two conserved domains: region I, at the $\mathrm{N}$-terminus of the repeats, and a known C-terminal cell-adhesive adhesive motif, termed type I thrombospondin repeat (TSR) [44]. Even though the repeat regions' function has not been made clear to date, it has been found that they are species-specific, low complexity and (in many cases) are responsible for the diversity between different parasite isolates [45]. It has been suggested that the presence of polymorphic repeats affects antibody affinity maturation, thus suppressing antibody response to critical epitopes or adjacent regions [46].

\section{Bioinformatics analysis of PvRON4 primary sequence}

The PvRON4 amino acid sequence was scanned for predicting different characteristics. VCG-1 PvRON4 had 


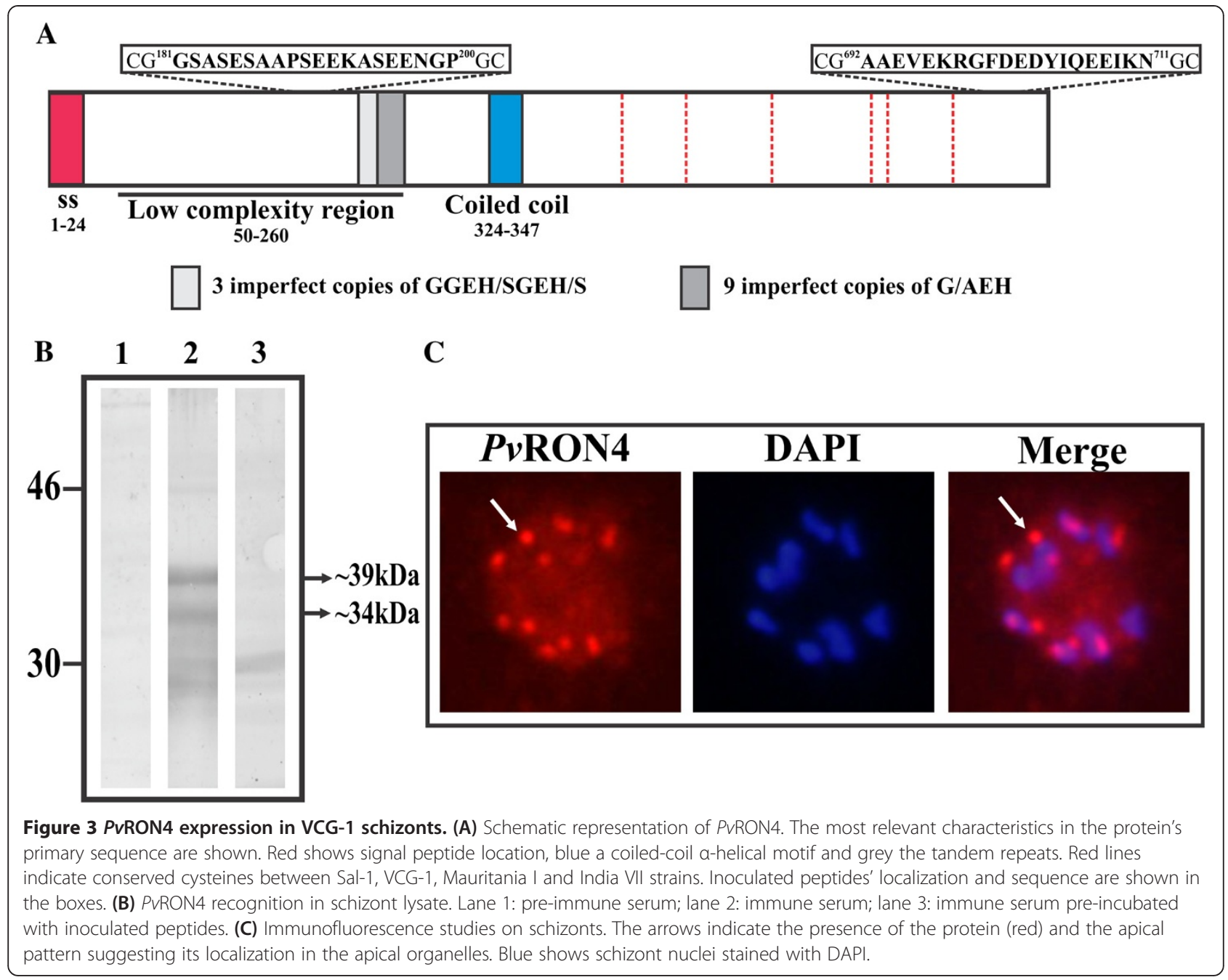

a signal peptide within the first 24 amino acids (Figure 3A) and a probable type I peptidase cleavage site between residues S24-F25, suggesting that once the protein has been synthesized, it is transported to be either secreted or inserted into cell membranes. In fact, it has been reported that PfRON4 and TgRON4 homologous proteins are secreted by the parasite towards host cell membrane $[47,48]$. Bioinformatics analysis detected no potential transmembrane domains or GPI anchors in PvRON4, TgRON4 and PfRON4, thereby classifying them as soluble proteins. It has been shown that some soluble $P$. falciparum merozoite surface proteins, such as MSP 3, 6, 7 and 9, are associated and/or interact with membrane-anchored antigens [49]. Interestingly, $P \nu$ RON4 contained a coiled-coil $\alpha$-helical motif in its sequence between residues 324-347 (Figure 3A) suggesting PvRON4 participation in protein-protein interaction and complex formation. A low complexity (Figure 3A), highly variable region was found (see Additional file 1) between residues
50 to 260 containing two TRs. The first repeat consisted of seven amino acids (GGEH/SGEH/S) and the second of three amino acids having nine imperfect copies of G/AEH residues. A conserved region between residues 277 and 733 was found when comparing RON4 from VCG-1 with other $P$. vivax strains, such region being characterized by having six conserved cysteines. Several functionally relevant malarial antigens, such as AMA-1 and RON2, have cysteine-containing regions forming disulphide bridges and/or ordered 3D structures for fulfilling their biological function [21]. However, further structural and functional studies are needed for evaluating this conserved domain's importance in PvRON4 and the influence of repeat regions in an immune response against $P v R O N 4$.

\section{PVRON4 expression and localization in VCG-1 late-stage} schizonts

Anti- $P \nu$ RON4 antibodies were induced in mice for evaluating $P v$ RON4 expression and cell localization in $P$. 
vivax schizonts. ELISA revealed that immune sera showed high reactivity against 35114 and 35115 peptides $\left(\mathrm{A}_{620} 0.63 \pm 0.22\right)$ compared to pre-immune sera $\left(\mathrm{A}_{620}\right.$ $0.03 \pm 0.01)(\mathrm{p}=0.00)$. Sera ability to recognize $P v \mathrm{RON} 4$ in VCG-1 schizont lysate was then evaluated using Western blot. Figure 3B shows that polyclonal antibodies specifically recognized $\sim 39 \mathrm{kDa}$ and $\sim 34 \mathrm{kDa}$ bands which were not recognized by either the pre-immune sera or hyper-immune sera which had been pre-incubated with the inoculated peptides. Taking a $\sim 80 \mathrm{kDa}$ predicted PvRON4 size, two bands below the expected molecular weight were detected, suggesting that PvRON4 undergoes a similar proteolytic processing to that reported for most proteins localized in the apical organelles, including other RON proteins [47]. It has been suggested that rhoptry proteins' proteolytic cleavage is an essential event for maturation and/or promoting interaction with cell surface receptors or between parasite proteins $[47,50]$.

The anti-PvRON4 antisera also recognized the protein on VCG-1 late-stage schizonts, having the typical apical organelle punctate pattern (Figure $3 \mathrm{C}$ ) common to other apical proteins $[8,18]$. Co-localization studies using antibodies against PvRON4 and $P v 12$ showed that while
Pv12 was expressed on merozoite surface with a bunch of grapes-like pattern, $P v$ RON4 had a punctate pattern, not overlapping $P v 12$ staining (Figure 4). When anti-PvRON4 antibodies were used with anti$P v$ RON2, the staining overlapped, coinciding with TgRON4 and PfRON4 localization in the rhoptry neck (Figure 4). Previous studies in T. gondii and P. falciparum have shown that RON4 is the only protein where strong evidence has been produced concerning localization in the TJ $[25,51]$ and has been chosen as a model for studying the dynamics of merozoite invasion of RBC [25].

\section{Conclusions}

Bioinformatics and experimental analyses allowed the identification of a new gene having three exons and encoding the PvRON4 protein. Like PfRON4, its P. falciparum homologue, $P v \mathrm{RON} 4$ is expressed in late schizonts and is localized in the rhoptries. Strong evidence regarding RON4 association in the TJ and its conservation in the Plasmodium genus has highlighted this protein's importance and the need for evaluating its mechanism of action and association within the P. vivax AMA1-RON complex.

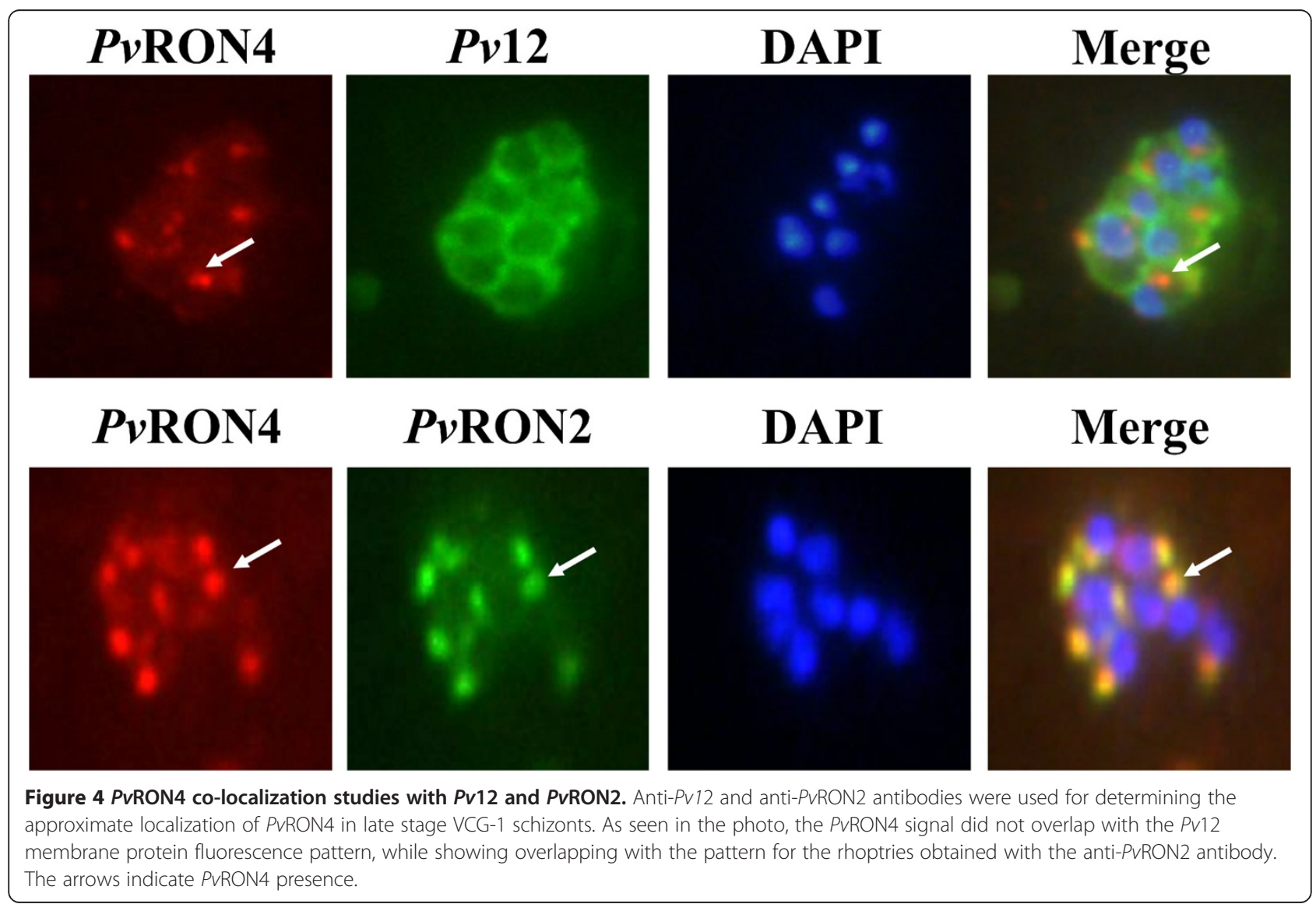




\section{Additional file}

Additional file 1: PvRON4 amino acid alignment between different Plasmodium vivax strains. Conserved cysteines between VCG-1 (Colombia), Sal-1 (Salvador), India VII (India) and Mauritania I (Africa) strains are shown in red.

\section{Competing interests}

The authors declare that they have no competing interests.

\section{Authors' contributions}

GAP carried out bioinformatics analyses, molecular biology assays and wrote the initial manuscript. HC synthesized and purified the peptides used for rabbit and mice immunizations and analyzed data. JA carried out immunoassays. MAP evaluated and coordinated assays, and revised the final manuscript. All authors read and approved the final manuscript.

\section{Acknowledgements}

We would like to thank Jason Garry for translating and reviewing this manuscript. This research was supported by the "Departamento Administrativo de Ciencia, Tecnología e Innovación (COLCIENCIAS)", contract RC\#0309-2013.

Received: 9 August 2013 Accepted: 2 October 2013

Published: 5 October 2013

\section{References}

1. WHO: World malaria report. Geneva: WHO Press; 2011

2. Price RN, Tjitra E, Guerra CA, Yeung S, White NJ, Anstey NM: Vivax malaria: neglected and not benign. Am J Trop Med Hyg 2007, 77:79-87.

3. Beg MA, Khan R, Baig SM, Gulzar Z, Hussain R, Smego RA Jr: Cerebral involvement in benign tertian malaria. Am J Trop Med Hyg 2002, 67:230-232.

4. Anstey NM, Russell B, Yeo TW, Price RN: The pathophysiology of vivax malaria. Trends Parasitol 2009, 25:220-227.

5. Baird JK: Resistance to therapies for infection by Plasmodium vivax. Clin Microbiol Rev 2009, 22:508-534.

6. Noulin F, Borlon C, Van Den Abbeele J, D'Alessandro U, Erhart A: 1912 2012: a century of research on Plasmodium vivax in vitro culture. Trends Parasitol 2013, 29:286-294.

7. Pico De Coana Y, Rodriguez J, Guerrero E, Barrero C, Rodriguez R, Mendoza M, Patarroyo MA: A highly infective Plasmodium vivax strain adapted to Aotus monkeys: quantitative haematological and molecular determinations useful for $P$. vivax malaria vaccine development. Vaccine 2003, 21:3930-3937.

8. Arevalo-Pinzon G, Curtidor H, Patino LC, Patarroyo MA: PvRON2, a new Plasmodium vivax rhoptry neck antigen. Malar J 2011, 10:60

9. Mongui A, Perez-Leal O, Rojas-Caraballo J, Angel DI, Cortes J, Patarroyo MA Identifying and characterising the Plasmodium falciparum RhopH3 Plasmodium vivax homologue. Biochem Biophys Res Commun 2007 358:861-866.

10. Moreno-Perez DA, Areiza-Rojas R, Florez-Buitrago X, Silva Y, Patarroyo ME, Patarroyo MA: The GPI-anchored 6-Cys protein Pv12 is present in detergent-resistant microdomains of Plasmodium vivax blood stage schizonts. Protist 2013, 164:37-48.

11. Moreno-Perez DA, Mongui A, Soler LN, Sanchez-Ladino M, Patarroyo MA: Identifying and characterizing a member of the RhopH1/Clag family in Plasmodium vivax. Gene 2011, 481:17-23.

12. Cowman AF, Berry D, Baum J: The cellular and molecular basis for malaria parasite invasion of the human red blood cell. J Cell Biol 2012, 198:961-971.

13. Baum J, Gilberger TW, Frischknecht F, Meissner M: Host-cell invasion by malaria parasites: insights from Plasmodium and Toxoplasma. Trends Parasitol 2008, 24:557-563.

14. Nichols BA, Chiappino ML, O'Connor GR: Secretion from the rhoptries of Toxoplasma gondii during host-cell invasion. J Ultrastruct Res 1983, 83:85-98

15. Stewart MJ, Schulman S, Vanderberg JP: Rhoptry secretion of membranous whorls by Plasmodium falciparum merozoites. Am J Trop Med Hyg 1986, 35:37-44.
16. Bradley PJ, Ward C, Cheng SJ, Alexander DL, Coller S, Coombs GH, Dunn JD, Ferguson DJ, Sanderson SJ, Wastling JM, Boothroyd JC: Proteomic analysis of rhoptry organelles reveals many novel constituents for host-parasite interactions in Toxoplasma gondii. J Biol Chem 2005, 280:34245-34258.

17. Cao J, Kaneko O, Thongkukiatkul A, Tachibana M, Otsuki H, Gao Q, Tsuboi T, Torii M: Rhoptry neck protein RON2 forms a complex with microneme protein AMA1 in Plasmodium falciparum merozoites. Parasitol Int 2009 58:29-35.

18. Morahan BJ, Sallmann GB, Huestis R, Dubljevic V, Waller KL: Plasmodium falciparum: genetic and immunogenic characterisation of the rhoptry neck protein PfRON4. Exp Parasitol 2009, 122:280-288.

19. Curtidor H, Patino LC, Arevalo-Pinzon G, Patarroyo ME, Patarroyo MA: Identification of the Plasmodium falciparum rhoptry neck protein 5 (PfRON5). Gene 2011, 474:22-28.

20. Collins CR, Withers-Martinez C, Hackett F, Blackman MJ: An inhibitory antibody blocks interactions between components of the malarial invasion machinery. PLoS Pathog 2009, 5:e1000273.

21. Vulliez-Le Normand B, Tonkin ML, Lamarque MH, Langer S, Hoos S, Roques M, Saul FA, Faber BW, Bentley GA, Boulanger MJ, Lebrun M: Structural and functional insights into the malaria parasite moving junction complex. PLoS Pathog 2012, 8:e1002755.

22. Srinivasan $P$, Beatty WL, Diouf A, Herrera R, Ambroggio X, Moch JK, Tyler JS, Narum DL, Pierce SK, Boothroyd JC, Haynes JD, Miller LH: Binding of Plasmodium merozoite proteins RON2 and AMA1 triggers commitment to invasion. Proc Natl Acad Sci USA 2011, 108:13275-13280.

23. Lamarque M, Besteiro S, Papoin J, Roques M, Vulliez-Le Normand B, MorlonGuyot J, Dubremetz JF, Fauquenoy S, Tomavo S, Faber BW, Kocken $\mathrm{CH}_{\text {, }}$ Thomas AW, Boulanger MJ, Bentley GA, Lebrun M: The RON2-AMA1 interaction is a critical step in moving junction-dependent invasion by apicomplexan parasites. PLoS Pathog 2011, 7:e1001276.

24. Giovannini D, Spath S, Lacroix C, Perazzi A, Bargieri D, Lagal V, Lebugle C, Combe A, Thiberge S, Baldacci P, Tardieux I, Menard R: Independent roles of apical membrane antigen 1 and rhoptry neck proteins during host cell invasion by apicomplexa. Cell Host Microbe 2011, 10:591-602.

25. Riglar DT, Richard D, Wilson DW, Boyle MJ, Dekiwadia C, Turnbull L, Angrisano F, Marapana DS, Rogers KL, Whitchurch CB, Beeson JG, Cowman AF, Ralph SA, Baum J: Super-resolution dissection of coordinated events during malaria parasite invasion of the human erythrocyte. Cell Host Microbe 2011, 9:9-20.

26. McBride JS, Heidrich HG: Fragments of the polymorphic Mr 185,000 glycoprotein from the surface of isolated Plasmodium falciparum merozoites form an antigenic complex. Mol Biochem Parasitol 1987, 23:71-84.

27. Straub KW, Cheng SJ, Sohn CS, Bradley PJ: Novel components of the Apicomplexan moving junction reveal conserved and coccidia-restricted elements. Cell Microbiol 2009, 11:590-603.

28. Burge C, Karlin S: Prediction of complete gene structures in human genomic DNA. J Mol Biol 1997, 268:78-94.

29. Spidey. http://www.ncbi.nlm.nih.gov/spidey/.

30. Nielsen $\mathrm{H}$, Engelbrecht J, Brunak S, von Heijne G: Identification of prokaryotic and eukaryotic signal peptides and prediction of their cleavage sites. Protein Eng 1997, 10:1-6.

31. Kall L, Krogh A, Sonnhammer EL: An HMM posterior decoder for sequence feature prediction that includes homology information. Bioinformatics 2005, 21(Suppl 1):i251-257.

32. Krogh A, Larsson B, von Heijne G, Sonnhammer EL: Predicting transmembrane protein topology with a hidden Markov model: application to complete genomes. J Mole Biol 2001, 305:567-580.

33. Pierleoni A, Martelli PL, Casadio R: PredGPI: a GPI-anchor predictor. BMC Bioinformatics 2008, 9:392.

34. Letunic I, Goodstadt L, Dickens NJ, Doerks T, Schultz J, Mott R, Ciccarelli F, Copley RR, Ponting CP, Bork P: Recent improvements to the SMART domain-based sequence annotation resource. Nucleic Acids Res 2002 30:242-244.

35. Chomczynski P: A reagent for the single-step simultaneous isolation of RNA, DNA and proteins from cell and tissue samples. Biotechniques 1993, 15:532-534. 536-537.

36. Moreno-Perez DA, Montenegro M, Patarroyo ME, Patarroyo MA Identification, characterization and antigenicity of the Plasmodium vivax rhoptry neck protein 1 (PVRON1). Malar J 2011, 10:314. 
37. Neafsey DE, Galinsky K, Jiang RH, Young L, Sykes SM, Saif S, Gujja S, Goldberg JM, Young S, Zeng Q, Chapman SB, Dash AP, Anvikar AR, Sutton PL, Birren BW, Escalante AA, Barnwell JW, Carlton JM: The malaria parasite Plasmodium vivax exhibits greater genetic diversity than Plasmodium falciparum. Nat Genet 2012, 44:1046-1050.

38. Thompson JD, Higgins DG, Gibson TJ: CLUSTAL W: improving the sensitivity of progressive multiple sequence alignment through sequence weighting, position-specific gap penalties and weight matrix choice. Nucleic Acids Res 1994, 22:4673-4680.

39. Geourjon C, Deleage G, Roux B: ANTHEPROT: an interactive graphics software for analyzing protein structures from sequences. J Mol Graph 1991, 9:188-190. 167.

40. Larsen JE, Lund O, Nielsen M: Improved method for predicting linear B-cell epitopes. Immunome Res 2006, 2:2.

41. Escalante AA, Ayala FJ: Phylogeny of the malarial genus Plasmodium derived from rRNA gene sequences. Proc Natl Acad Sci USA 1994, 91:11373-11377.

42. Bozdech Z, Llinas M, Pulliam BL, Wong ED, Zhu J, DeRisi JL: The transcriptome of the intraerythrocytic developmental cycle of Plasmodium falciparum. PLOS Biol 2003, 1:E5.

43. Florens $L$, Washburn MP, Raine JD, Anthony RM, Grainger M, Haynes JD, Moch JK, Muster N, Sacci JB, Tabb DL, Witney AA, Wolters D, Wu Y, Gardner MJ, Holder AA, Sinden RE, Yates JR, Carucci DJ: A proteomic view of the Plasmodium falciparum life cycle. Nature 2002, 419:520-526.

44. Dame JB, Williams JL, McCutchan TF, Weber JL, Wirtz RA, Hockmeyer WT, Maloy WL, Haynes JD, Schneider I, Roberts D, Sanders GS, Reddy EP, Diggs $\mathrm{CL}$, Miller LH: Structure of the gene encoding the immunodominant surface antigen on the sporozoite of the human malaria parasite Plasmodium falciparum. Science 1984, 225:593-599.

45. Fenton $B$, Walker A, Walliker D: Protein variation in clones of Plasmodium falciparum detected by two dimensional electrophoresis. Mol Biochem Parasitol 1985, 16:173-183.

46. Hisaeda H, Yasutomo K, Himeno K: Malaria: immune evasion by parasites. Int J Biochem Cell Biol 2005, 37:700-706.

47. Besteiro S, Michelin A, Poncet J, Dubremetz JF, Lebrun M: Export of a Toxoplasma gondii rhoptry neck protein complex at the host cell membrane to form the moving junction during invasion. PLoS Pathog 2009, 5:e1000309.

48. Proellocks NI, Coppel RL, Waller KL: Dissecting the apicomplexan rhoptry neck proteins. Trends Parasitol 2010, 26:297-304.

49. Kauth CW, Woehlbier U, Kern M, Mekonnen Z, Lutz R, Mucke N, Langowski J, Bujard H: Interactions between merozoite surface proteins 1, 6, and 7 of the malaria parasite Plasmodium falciparum. J Biol Chem 2006, 281:31517-31527.

50. Pachebat JA, Kadekoppala M, Grainger M, Dluzewski AR, Gunaratne RS, Scott-Finnigan TJ, Ogun SA, Ling IT, Bannister LH, Taylor HM, Mitchell GH, Holder AA: Extensive proteolytic processing of the malaria parasite merozoite surface protein 7 during biosynthesis and parasite release from erythrocytes. Mol Biochem Parasitol 2007, 151:59-69.

51. Lebrun M, Michelin A, El Hajj H, Poncet J, Bradley PJ, Vial H, Dubremetz JF: The rhoptry neck protein RON4 re-localizes at the moving junction during Toxoplasma gondii invasion. Cell Microbiol 2005, 7:1823-1833.

\section{doi:10.1186/1475-2875-12-356}

Cite this article as: Arévalo-Pinzón et al:: Annotation and characterization of the Plasmodium vivax rhoptry neck protein 4 (PvRON4). Malaria Journal 2013 12:356.

\section{Submit your next manuscript to BioMed Central and take full advantage of:}

- Convenient online submission

- Thorough peer review

- No space constraints or color figure charges

- Immediate publication on acceptance

- Inclusion in PubMed, CAS, Scopus and Google Scholar

- Research which is freely available for redistribution 